\title{
Cosmic Rays (gammas, hadrons, neutrinos)
}

\author{
Thomas Lohse ${ }^{* \dagger}$ \\ Humboldt University Berlin, Germany \\ E-mail: lohse@ifh.de
}

\begin{abstract}
Almost a century after their dicovery by Victor Hess, the origin of cosmic rays is still far from understood. The composition and spectral behaviour of cosmic rays at very high energies is not well known, the cosmic ray sources have not yet been convincingly identified and experimental information is insufficient to fully understand the acceleration of cosmic rays in source candidates and the propagation through the interstellar and intergalactic medium. Also, dark matter annihilation, the decay of superheavy relic-particles from the big bang and other exotic origins are possibly responsible for at least part of the cosmic rays. Very high energy cosmic rays are measured directly by detection of the extensive air showers they induce when interacting in the atmosphere. Source locations and acceleration mechanisms, however, can best be revealed by measuring high energy photons or neutrinos which are thought to be produced in interactions of charged cosmic rays in the ambient medium of the sources. A broad experimental program is ongoing, exploiting various kinds of messenger particles, hadrons, gamma rays, and neutrinos. The status and recent results will be reviewed.
\end{abstract}

International Europhysics Conference on High Energy Physics

July 21st - 27th 2005

Lisboa, Portugal

\footnotetext{
${ }^{*}$ Speaker.

${ }^{\dagger}$ Supported by the German Ministry for Education and Research (BMBF)
} 


\section{Introduction}

Charged cosmic rays consist mostly of protons and heavier nuclei with a small admixture of antiprotons, electrons and positrons. Almost a century of research has led to a precise measurement of the cosmic ray flux for an energy range spanning more than 10 orders of magnitude, over which the flux varies by more than 30 orders of magnitude, compare Fig. 1 (from [1]). Above energies of $10 \mathrm{GeV}$, where the flux is not any longer influenced by the local environment like the magnetic field of the earth modulated by the solar activity, the energy spectrum of cosmic rays exhibits an almost power-law like shape, $d N / d E \propto$ $E^{-\alpha}$ and is hence clearly non-thermal. Power-law spectra can be naturally explained by dynamical processes, most importantly the acceleration of charged particles in plasma shockwaves as formed in many violent processes in the universe, as in supernova explosions or in relativistic plasma jets emitted from accreting black holes.

At energies around $10^{15} \mathrm{eV}$, the so-called "knee", the cosmic ray spectrum steepens, the spectral index $\alpha$ increasing from 2.7 to 3.1 . While $80 \%$

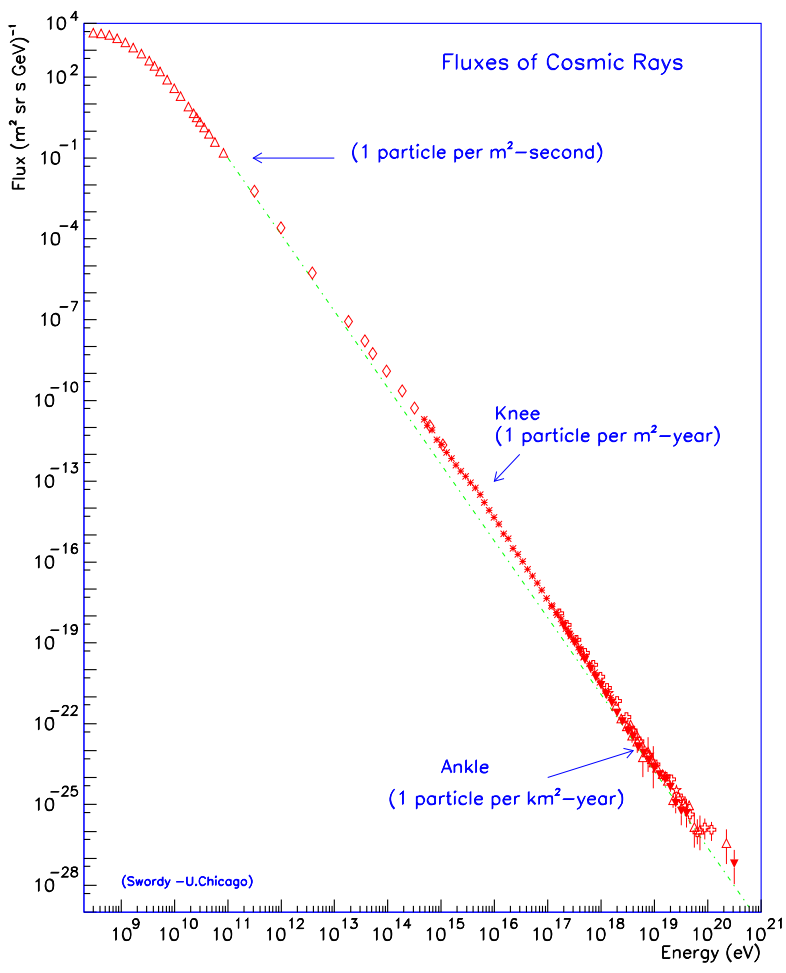

Figure 1: Flux of cosmic rays as function of energy [1]. of the cosmic rays below the knee are protons, all recent experiments agree that the mean atomic mass increases at the knee, probably making iron nuclei most abundant above the knee. At energies above $10^{18} \mathrm{eV}$ the spectrum seems to become harder again, a structure known as the "ankle" which may be associated with a transition to a dominating harder spectrum of extragalactic cosmic rays. Experimental information in this energy range, where the flux is extremely small, is still scarce and controversial.

In spite of the overall impressive achievements in measuring cosmic ray fluxes, we are still far from completely understanding the physics of cosmic rays. What is causing the knee and the ankle? What is the origin of cosmic rays beyond the ankle and which particles are they composed of? What and where are the sources of cosmic rays and how do the acceleration mechanisms work? How are the high energy particles transported through the interstellar and intergalactic medium? Is there a non-accelerated cosmic ray component due to the decay of super-heavy exotic particles?

In order to answer these questions it is not suffi cient to study charged cosmic rays alone, since these are deflected by interstellar and intergalactic magnetic fi elds and hence carry no information about source directions. An exception are the highest energy particles above the ankle which, depending on the not well known strength of the intergalactic magnetic fi elds, might be only mildly 
deflected. High energy gamma rays and neutrinos, however, are ideal messenger particles which reach the earth undeflected by magnetic fi elds.

High energy gamma rays are produced in the decay of neutral pions, which are created in the interaction of cosmic ray protons or nuclei with the ambient medium of the sources. Gamma rays are relatively easy to detect and gamma ray astronomy is fully established since more than a decade. However, gamma ray emission by itself does not represent an unambiguous signature for cosmic rays, which are dominated by protons and nuclei, i.e. hadrons. Electrons and positrons, also expected to be accelerated to high energies, can as well produce gamma rays by inverse Compton scattering on the ambient radiation fi elds or, depending on the matter density in the source, by bremsstrahlung. The separation of hadronic and electronic production of gamma rays is a major challenge for gamma ray astronomy. In addition, the extragalactic background light makes the intergalactic medium oblique to highest energy gamma rays so that only relatively close-by sources are observable. This seeming disadvantage offers, however, the possibility to measure these radiation fields which are not well known and contain information about galaxy formation in the evolving universe.

Neutrinos are ideal messenger particles, since they are clearly connected to interaction of high energy hadrons in which secondary mesons produce neutrinos in their decays. The intergalactic medium is almost transparent to neutrinos so that sources at very large distances are accessible. Moreover, while gamma rays may be re-absorbed in the sources, neutrinos allow an unobscured view into the interior of sources. On the other hand, neutrinos are hard to detect. Neutrino astrophysics requires the construction of huge detectors exploiting new experimental techniques. While fully established for gamma rays, neutrino astronomy is still in the development stage.

At energies around the knee and above, fluxes of cosmic rays are too small to be detected by satellite or balloon experiments. Therefore, ground based detectors with large effective areas have to be used. The most important experimental techniques for ground based observation of charged cosmic rays, gamma rays and neutrinos are sketched in Fig. 2. Traditionally, extensive air showers (EAS) induced by cosmic ray hadrons or gamma rays are sampled by surface detector arrays

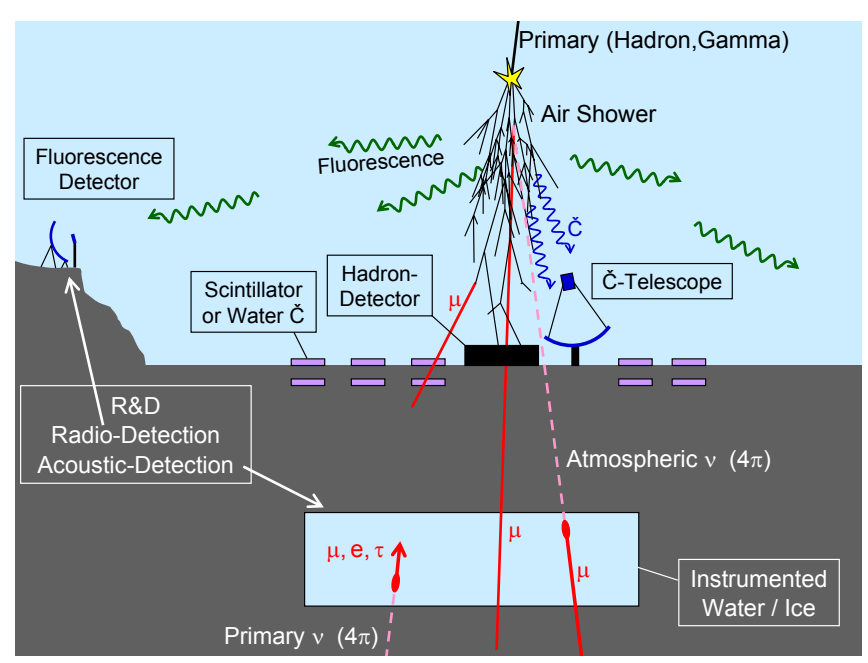

Figure 2: Sketch of experimental techniques for groundbased detection of high energy charged cosmic rays, gamma rays and neutrinos. consisting of scintillators or water Cherenkov detectors. Shielded detectors are used to identify muons from the decay of mesons in hadronic showers. Additional deep underground water or ice Cherenkov detectors can identify muons of higher energies. Specialized detectors, like calorimeters, are used to measure local energy fluxes. Showers can also be measured calorimetrically by collecting the fluorescence light from atmospheric nitrogen, excited by shower particles, in distant wide angle detectors. Recently, detection of radio emission from air showers has been 
achieved $[2,3]$, possibly opening a new and economic alternative to fluorescence detection for future experiments. Precise shower reconstruction at low energy thresholds is possible using imaging Cherenkov telescopes, which detect the Cherenkov light emitted in a narrow cone around the shower. High sensitivity, good angular resolution and high separation power between gamma ray showers and hadronic showers make them the currently most powerful instruments for ground based gamma ray astronomy. Finally, cosmic neutrinos are detected by their interaction in instrumented deep-underground water or ice volumes. Muons, produced by interaction of muon neutrinos, and showers ("cascades"), produced by electron and tau neutrinos, are detected by measuring the emitted Cherenkov light. In addition, radio and acoustic detection techniques for neutrino interactions are under development (for a review see [4]). Background of atmospheric muons can be eliminated by restricting the fi eld of view for muon neutrinos to the lower hemisphere, such as to use the earth as shielding. Nevertheless, the background of atmospheric neutrinos due to meson decays in air showers prevails. This background is, however, expected to become negligible at suffi ciently high energies, since atmospheric neutrinos are secondary particles and have a much steeper energy spectrum than the primary cosmic rays (and thus presumably the cosmic neutrinos).

\section{Cosmic rays around the knee}

In spite of the enormous experimental efforts of the last fi ve decades, the origin of the knee is not yet understood. It is generally believed that the knee is of astrophysical origin. At energies above the knee, accelerated particles might escape the galactic accelerators, like supernova shells or pulsar wind nebulae, since their gyro-radius in the local magnetic fi elds starts to exceed the source dimensions, or the diffusive losses of cosmic rays from our galaxy might increase at knee energies. Since in such scenarios the position of the knee is connected to the rigidity of cosmic rays in the interstellar magnetic fi elds, one would expect the knee energy to be different for different nuclei and scale with the charge number $Z$. However, also new particle physics, like the onset of a new kind of hadronic interactions influencing the transport through the interstellar medium or the interaction in the atmosphere, is conceivable. In the latter case one would rather expect the knee energy for individual nuclei to scale with the mass number $A$. A measurement of the energy spectrum around the knee for individual nuclei or at least individual mass groups of nuclei is necessary to test these scenarios.

Measuring the energy and nuclear mass of cosmic rays with EAS detectors is a notoriously diffi cult task and requires a detailed understanding of the development of air showers which are induced by very high energy nuclear interactions [5]. Presently, the KASCADE detector [6] and its extension KASCADE-Grande [7] in Karlsruhe, Germany, are used to perform such measurements at energies around the knee. The detector components, shielded and unshielded scintillation counters, providing information on electron and muon densities in the showers, augmented by muon tracking detectors and a central iron sampling calorimeter to measure the hadronic shower core, provide a broad variety of redundant information on individual showers. Using the fact that the ratio of muon and electron densities increases in a characteristic way with the nuclear mass, the nuclear composition can in principle be inferred, provided that the structure of air showers is well enough understood. The KASCADE collaboration developed a sophisticated unfolding algorithm [8] which allows to induce the spectra of individual mass groups, the "typical" nuclei of 

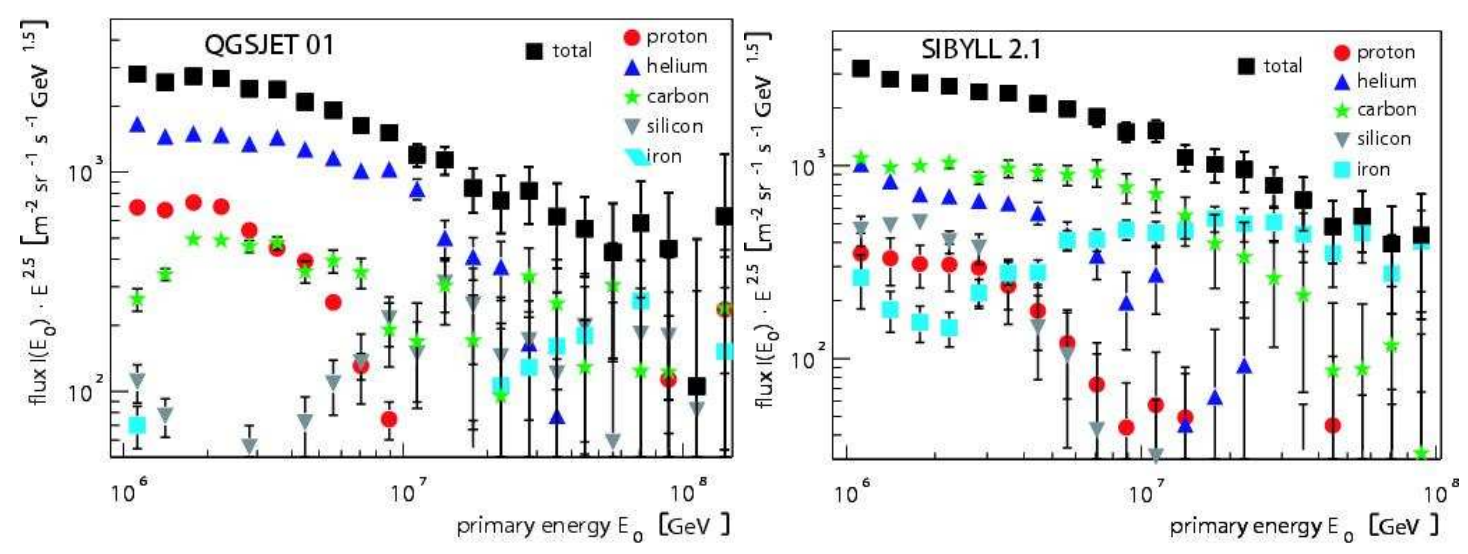

Figure 3: Unfolded fux measurement for individual element groups around the knee using two different hadronic interaction models for shower simulation (left: QGSJET, right: SIBYLL) [9].

which being protons, helium, carbon, silicon and iron, compare Fig. 3. Two different hadronic interaction models, QGSJET01 [10] and SIBYLL 2.1 [11], are used as input for the unfolding. As can be seen from Fig. 3, although the average nuclear mass is always found to increase around the knee, the detailed nuclear composition derived from the two models is very different. A detailed confrontation of the interaction models with the various shower observables accessible to KASCADE revealed that none of the models is able to satisfactorily describe all data. Further tuning of the models on the basis of the rich and redundant information on the structure of extensive air showers is under way. In addition, further input is needed from high energy hadronic interactions at accelerators, like the LHC.

\section{Cosmic rays beyond the ankle}

The highest energy cosmic rays beyond the ankle represent one of the most interesting puzzles in cosmic ray physics. It is generally believed that such particles are accelerated in the most violent astrophysical environments like the relativistic jets emitted by active galactic nuclei (AGN), supermassive accreting black holes at the centre of distant galaxies. However, once accelerated, ultrahigh energy protons and nuclei interact with the cosmic microwave background radiation (CMBR). Nuclei are destroyed by photo-disintegration so that highest energy cosmic rays are expected to be dominated by protons. Protons in turn lose energy while propagating through the CMBR field, the most important loss mechanism being photo-pion production with a threshold energy around $6 \times 10^{19} \mathrm{GeV}$. Therefore, one expects a cut-off in the cosmic ray spectrum at this energy, the socalled Greisen-Zatsepin-K'uzmin (GZK) cut-off [12, 13]. Should this cut-off be absent, the sources of the highest energy cosmic rays would have to be very close-by, at distances not further than a few tens of Mpc, or the cosmic rays would have to be weakly interacting particles, like neutrinos. Due to the low cross-sections for interaction in the earth's atmosphere, the latter scenario would, however, imply unrealistically high luminosities for the cosmic ray sources $[14,15]$.

Air showers with energies above $10^{20} \mathrm{eV}$ have been observed by several experiments, and their structure seems to indicate that cosmic rays above the ankle are indeed dominated by light nuclei, presumably protons. The spectral shape for the highest energy cosmic rays is, however, still unclear. 
While the air shower array AGASA measured a power law spectrum extending beyond $10^{20} \mathrm{GeV}$ without an apparent cutoff [16], evidence for a GZK-like cut-off was found with the fluorescence detector HiRes [17, 18], compare Fig. 4. Because of the steepness of the spectrum, this discrepancy could easily be accounted for by a $\approx 25 \%$ sytematic error in the energy scales of the experiments. In fact the energy determination is very different for fluorescence detectors, relying on good knowledge of the fluorescence yield and atmospheric properties, and ground arrays, depending more strongly on shower simulation and assumptions about the composition of primary cosmic rays.

It is expected that these problems will be solved at the Pierre Auger Observatory [21, 22] which is under construction in Argentina. AUGER is a hybrid detector, combining a

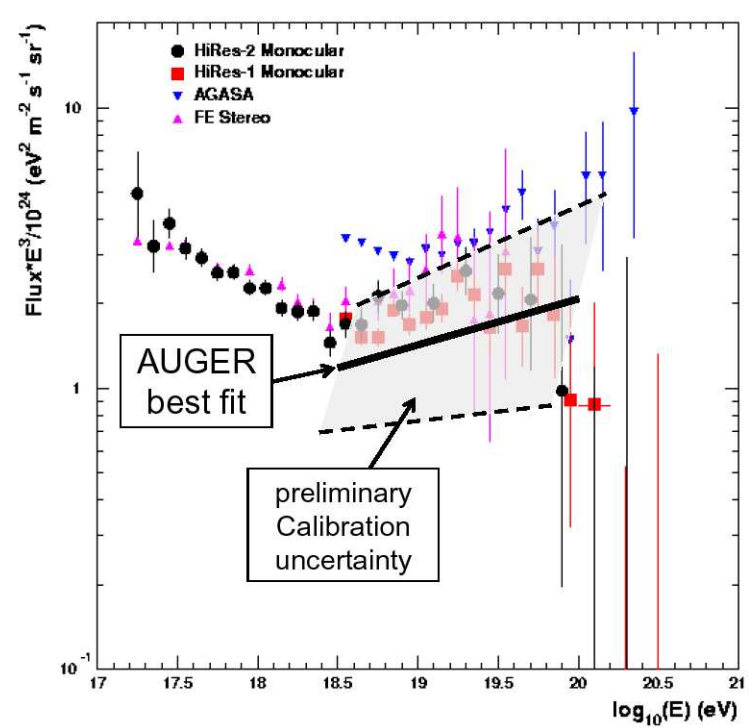

Figure 4: $\quad$ Energy flux (multiplied by $E^{3}$ ) of highest energy cosmic rays. Data points are from AGASA [16], HiRes [17] and Fly's Eye [19]. Also indicated is the best fit of the first AUGER data [20], together with a rough estimate of the present level of systematic uncertainties. Picture adapted from [17].

$3000 \mathrm{~km}^{2}$ surface array of 1600 water Cherenkov tanks with 4 fluorescence sites, each equipped with 6 telescopes. Presently more than 50\% of the surface detectors and 3 flourescence sites are operational. From Jan. 2004 until Jan. 2005 the continuously growing detector was used to accumulate an exposure for the surface detectors of $1750 \mathrm{~km}^{2} \mathrm{sr} y \mathrm{r}$, already slightly larger than that obtained by AGASA. The energy measured with the surface detectors was cross-calibrated with the model-independent calorimetric measurement from the fluorescence detectors in clean hybrid events, albeit with still signifi cant systematic errors due to the limited statistics of hybrid events available so far, the uncertainty in the fluorescence yield and the detector calibration uncertainties. The preliminary spectrum $(E \times d N / d E)$ obtained in this way is shown in Fig. 5. It can be fit by a power law $\left(\chi^{2} /\right.$ d.o.f. $\left.=2.4\right)$ which is indicated in Fig. 4 together with a rough estimate of the systematic errors (from an interpolation of the systematic errors given in Fig. 5). It can be seen that AUGER confi rms the spectral slope measured by the earlier measurements but is at this early stage not yet able to distinguish between the different normalizations. It is interesting to note, however, that not a single event with energy larger than $10^{20} \mathrm{eV}$ has yet passed the selection criteria.

Besides the spectral shape, also anisotropies in the arrival directions of highest energy cosmic rays are under dispute. The AGASA experiment reports clustering of events with energies above $4 \times 10^{19} \mathrm{eV}$ at a $2.5^{\circ}$ angular separation scale. Five doublets and one triplet were found. The chance probability of this signal was estimated to be $10^{-4}$ [23]. Non-uniform arrival directions around $10^{18} \mathrm{eV}$, suggestive of a source direction towards the Galactic centre, was reported by AGASA [24] and, less statistically signifi cant and in a slightly different direction, by SUGAR [25]. HiRes does not confi rm any of these signals [26, 18]. The AUGER collaboration, using an exposure larger than that accumulated by AGASA, searched for anisotropies for various angular scales and energy ranges and in addition for excess fluxes for candidate source directions 


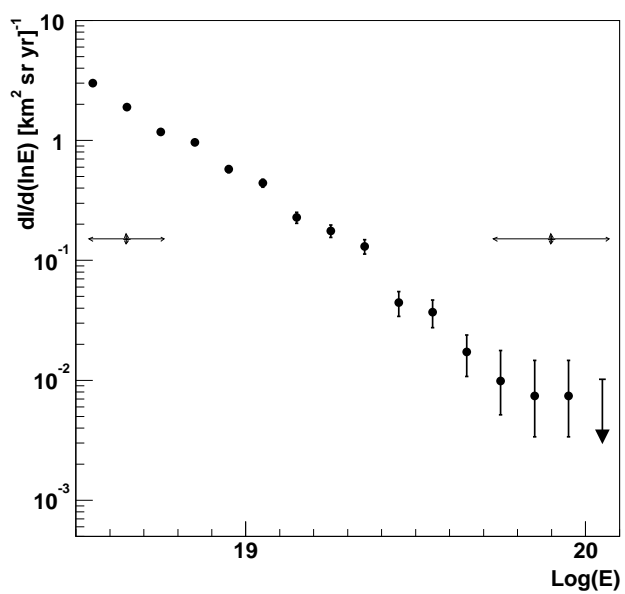

Figure 5: Initial cosmic ray spectrum measured by AUGER [20]. Error bars are statistical. The arrow is a 95\% CL upper limit. Systematic errors are indicated by double errors at two different energies.

(fi xed a priori) [27, 28, 22], and found no signifi cant signals. It thus appears more and more likely that the signals reported earlier were statistical fluctuations and that there are no anisotropies on the present level of sensitivity.

\section{Gamma rays from cosmic ray sources}

Very high energy gamma ray astronomy is witnessing a major scientific break-through, mostly due to the advent of a new generation of imaging Cherenkov telescopes. MAGIC [29] and VERITAS [30], the latter still in the construction phase, are exploring mostly the northern sky, while CANGAROO-III [31] and H.E.S.S. [32] are covering the south. The number of sources in the $\mathrm{TeV}$ range has roughly tripled in the last 2 years and much more is to be expected with the detectors coming up to speed. H.E.S.S. started full operation in December 2003, considerably earlier than the other detectors, and thus contributed most of the new results. Besides diffuse emission from several parts of the galactic plane, a large variety of galactic and extragalactic source classes has been established, like supernova explosion shells, nebulae powered by the winds of energetic pulsars, pulsars in binary systems, stellar mass and super-massive accreting black holes and mysterious sources of yet unknown nature. Only a subset of sources, most relevant in the context of the origin of cosmic rays will be discussed here; for a recent more complete review see [33].

Supernova explosions are prime candidates for cosmic ray sources. A conversion of about $10 \%$ of their mechanical energy suffi ces to sustain the observed Galactic cosmic ray population. An appropriate acceleration mechanism is diffusive shock acceleration in expanding explosion shells (for a recent review see [34]). Two supernova shells, both originally discovered as TeV gamma ray

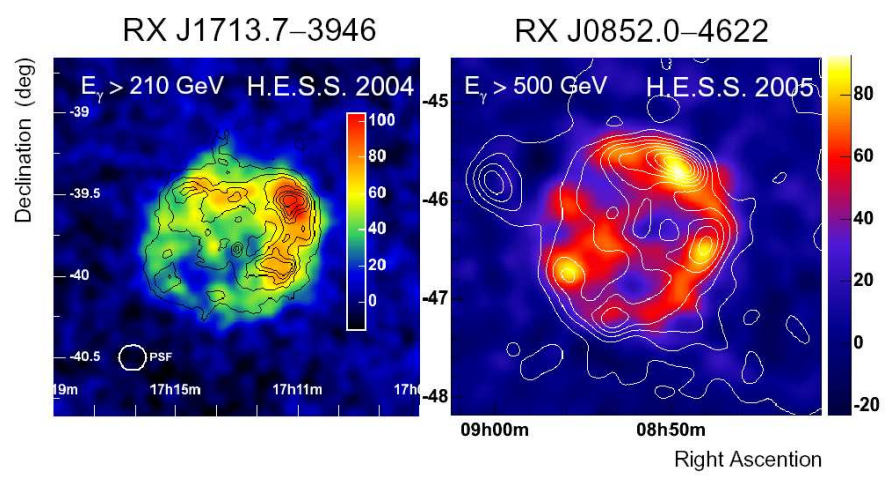

Figure 6: Gamma ray count maps for the supernova remnants RX J1713.7-3946 (left) [37, 38] and RX J0852.0-4622 (right) $[39,40]$ from H.E.S.S.. Contour lines indicate the X-ray emission from ASCA (left) [41] and ROSAT (right) [42]. sources by CANGAROO [35, 36], were recently spatially resolved in $\mathrm{TeV}$ gamma rays by H.E.S.S. [37, $38,39,40]$, see Fig. 6. The morphologies are well correlated with those seen in X-rays and shellstructures are visible. The spectrum of the supernova remnant RX J1713.7-3946 (Fig. 7) was measured up to $30 \mathrm{TeV}$ and approximately follows a power law of index 2.1 to 2.3 with an indication of a high-energy cut-off. 

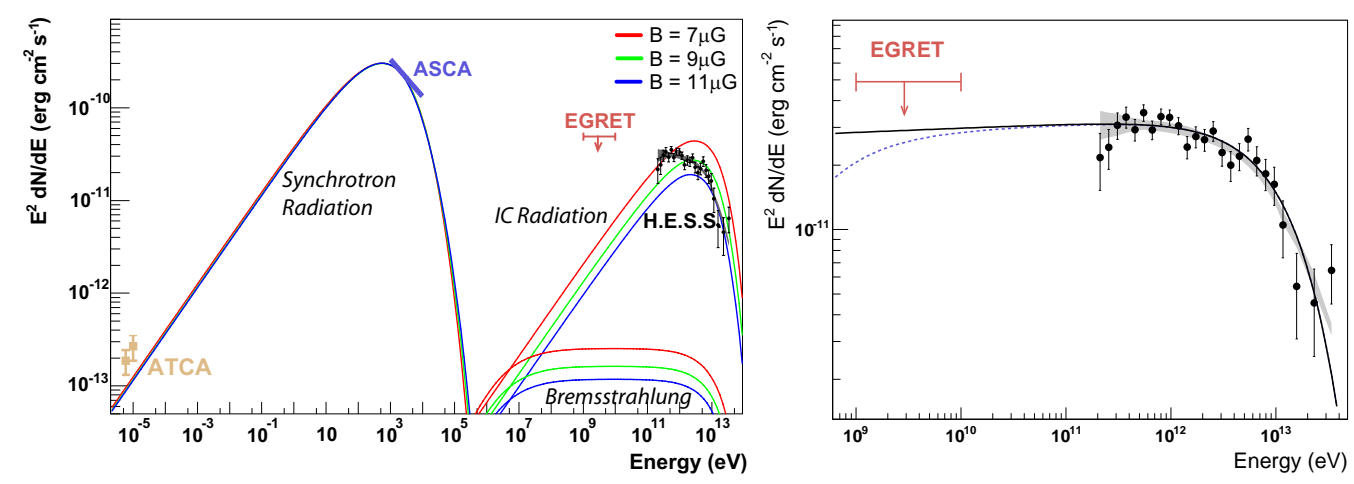

Figure 7: Left: Broadband spectral energy density of RX J1713.7-3946 together with model predictions for an electronic origin of the high energy gamma rays for three different values of the mean magnetic field in the emission region. The electron luminosity is adjusted such that the measured X-ray flux is well described. Right: Fit of the high energy end of the spectrum by a power law with exponential cut-off (solid line), as expected for hadronic scenarios. The dashed line is a modification which takes the $\pi^{0}$ production kinematics into account. The shaded band indicates the systematic errors for the data points.

While these observations confi rm the acceleration of charged particles to energies of $10 \mathrm{TeV}$ and beyond, it is much more diffi cult to show that the shells are indeed accelerating nuclei and not only electrons. Fig. 7 shows the result of simple model fi ts to the H.E.S.S. data. The broad band spectral energy density (left) shows a double hump structure. Synchrotron radiation of electrons in the local magnetic fi elds leads to a broad emission spectrum ranging from the radio to the X-Ray regime. This structure can be used to $f i x$ the magnetic fi eld strength for a given spectrum of accelerated electrons, the latter normally assumed to be a power law with index $\approx 2$ with an exponential cut-off around $100 \mathrm{TeV}$. The emission of very high energy gamma rays is then explained by the same electron population undergoing inverse Compton scattering with the (known) lower energy radiation fi elds, like ambient infrared light, the cosmic microwave background, or the synchrotron radiation itself. The normally much less important bremsstrahlung contribution to the very high energy gamma rays can also be predicted if estimates for the matter density are used. Modest variations of the magnetic fi eld can be compensated in such models by an adjustment of electron luminosities or mean energies, as to keep the synchrotron spectrum stable. Changing the electron spectrum, however, affects the very high energy emission. This can be seen in Fig. 7 (left). It is found that no satisfactory fi $t$ of the H.E.S.S. data is possible. In addition the magnetic fi elds which are required to match the integrated gamma ray flux is much lower than expected for compressed shells which exhibit very sharp structures in high resolution X-ray observations [43]. At much higher magnetic fi elds, however, the inverse Compton and bremsstrahlung contributions would become negligibly small. A better description of the data is possible with spectral shapes expected from hadronic models in which high energy nuclei interact with ambient matter and radiation fi elds and produce neutral pions, see Fig. 7 (right). Contrary to the electronic production, this process is not linked to the synchrotron emission.

Although the nucleonic origin seems more plausible in these simple models, it has to be kept in mind that the strong correlation of X-ray and gamma ray intensities indicates a contribution from electrons. In fact, in hadronic scenarios, a high correlation with the CO-radio emission from closeby molecular clouds is expected. Such correlations are indeed found [38] but they are much less striking than the correlation with X-rays. In summary, in spite of enormous progress, supernova 
remnants are not yet unambiguously identifi ed as accelerators of cosmic ray nuclei.

Another interesting TeV gamma ray source is the Galactic centre region, fi rst detected by CANGAROO [44] and VERITAS [45] and then detected with much higher precision and angular resolution by H.E.S.S. [46, 47, 48]. H.E.S.S. reported a much harder spectrum than CANGAROO and re-observations revealed no signs of variability. MAGIC has recently also detected the Galactic centre region and confi rmed the H.E.S.S. spectrum [29]. Within the present systematic pointing error of $20^{\prime \prime}$, the location of the H.E.S.S. source is compatible with the black hole, Sgr A*, but an origin in the close-by supernova remnant Sgr A East cannot be excluded yet. Astrophysical models for particle acceleration exist for both objects, but also the annihilation of speculative dark matter particles, the density of which is expected to peak at the Galactic centre, might explain at least part of the signal. Fig. 8 compares the H.E.S.S. spectrum with two specifi $\mathrm{c}$ dark matter models.

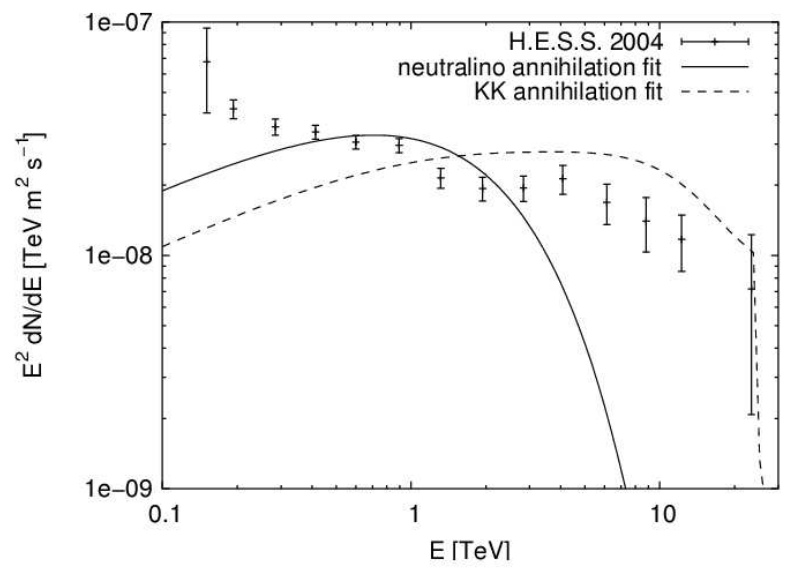

Figure 8: Spectral energy density of gamma rays from the Galactic centre source HESS J1745-290 together with model predictions for spectra from annihilation of $20 \mathrm{TeV}$ MSSM neutralinos and from $20 \mathrm{TeV}$ KaluzaKlein dark matter particles; picture from [48].

Independently of the model, the absence of a cut-off in the spectrum up to energies of 10 to $20 \mathrm{TeV}$ implies, due to energy conservation, a mass of the hypothetic particles much larger than preferred in most models. In addition, fi tting the high energy end with dark matter annihilation models leaves a very peculiar gamma ray spectrum at lower energies to be explained by astrophysical processes. It is hence unlikely that the signal from the Galactic centre is dominated by dark matter annihilation, though partial contributions (especially at lower energies) are not excluded.

In summer 2004 H.E.S.S. started a systematic survey of the galactic plane by scanning a region of $\pm 30^{\circ}$ in Galactic longitude and $\pm 3^{\circ}$ in latitude. This effort brought a rich harvest (see Fig. 9) [49, 50, 51]: Besides three known sources, the Galactic centre and the super-

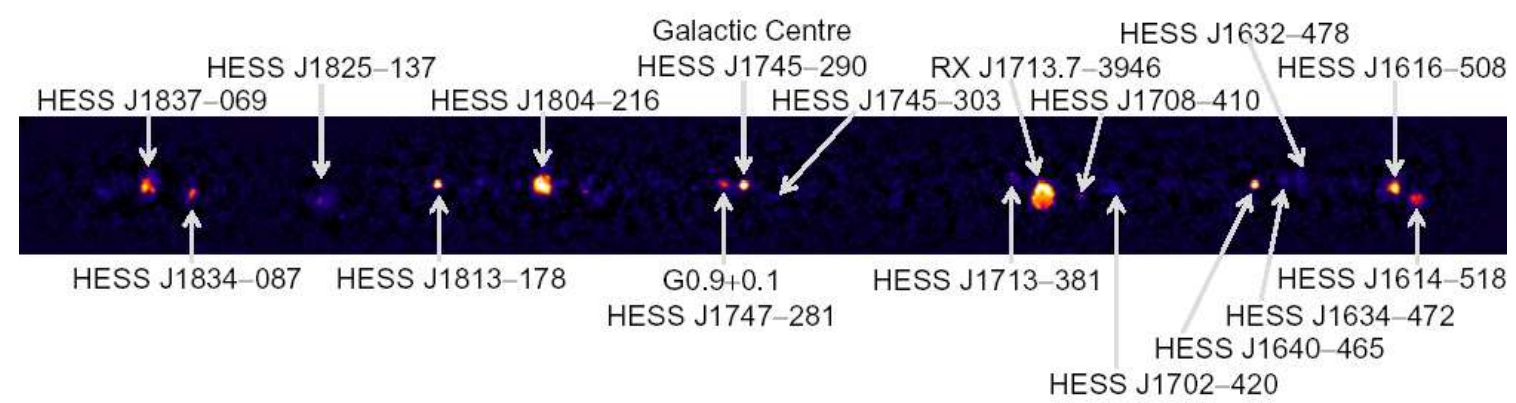

Figure 9: Significance map for the H.E.S.S. survey of the inner galactic plane, covering $\pm 30^{\circ}$ in galactic longitude around the Galactic centre. The typical energy threshold for the scan is $250 \mathrm{GeV}$. The significance of the indicated sources ranges from 4 to almost 30 standard deviations. 
nova remnants G0.9+0.1 and RX J1713.7-3946, 15 new sources were discovered, 14 of which are indicated in Fig. 9. They exhibit hard power-law spectra, with indices ranging form 1.8 to 2.7, as expected for shock-wave accelerators. Almost all sources are extended, confi rming their galactic origin. Counterparts have been searched in other wavebands. Five sources could be related to supernova remnants, several other sources might be associated to nebulae powered by energetic pulsars. At least three seem to have no counterpart in any other waveband. Such sources are often called "dark-accelerators". They represent a still mysterious class of cosmic accelerators of totally unknown nature. Before the H.E.S.S. survey, two such sources had already been discovered serendipitously in observations of other targets, the fi rst one by HEGRA [53] and the second by H.E.S.S. [54]. Especially the absence of an X-ray counterpart makes these sources very interesting, since this might hint at a hadronic origin of the gamma ray signal.

The $15^{\text {th }}$ source detected in the H.E.S.S. survey (HESS J1826-148, close to HESS J1825-137 in Fig. 9) is coincident with the micro-quasar candidate LS 5039 [52], a few solar mass black hole in a 4 day orbit around a massive star. It represents a new class of gamma ray sources. The black hole accretes matter from the companion and emits two high energy plasma jets. Micro-quasars are galactic (i.e. close-by) stellar mass equivalents of active galactic nuclei (AGN) and might shed more light on the acceleration mechanisms in these extremely violent objects.

AGN are super-massive black holes accreting matter from the surrounding galaxy and emitting highly relativistic plasma jets. Up to now they represent the only extragalactic source class detected in $\mathrm{TeV}$ gamma rays. With one exception (the giant radio galaxy M87) all these AGN are so-called "blazars", for which the jet emission is almost aligned with the line of sight, leading to boosts of gamma ray energies and fluxes. AGN emission of $\mathrm{TeV}$ gamma rays is known to show strong variability down to time scales of hours and less, demonstrating that the sources have to be very compact. Black holes are the only known candidates. Spectral energy densities of AGN exhibit a two bump structure much alike that discussed for the supernova remnant RX J1713.7-3964 and both electronic and hadronic models have been developed to explain the spectra; for a recent review see [55].

Contrary to galactic sources, gamma rays from distant AGN suffer absorption in the extragalactic background light (EBL) leading to a steepening of the high energy end of the observed spectra. This offers a powerful method to constrain the not well known EBL density which can be related to cosmological parameters [56]. Fig. 10 shows the gamma ray horizon (the distance, expressed in redshift $z$, at which the gamma ray intensity at a given energy is reduced by a factor $e^{-1}$ ) versus energy as predicted by EBL models. The redshifts of the signifi cantly detected blazars [55] are indicated as well, ranging up to 0.186 . The energy thresh-

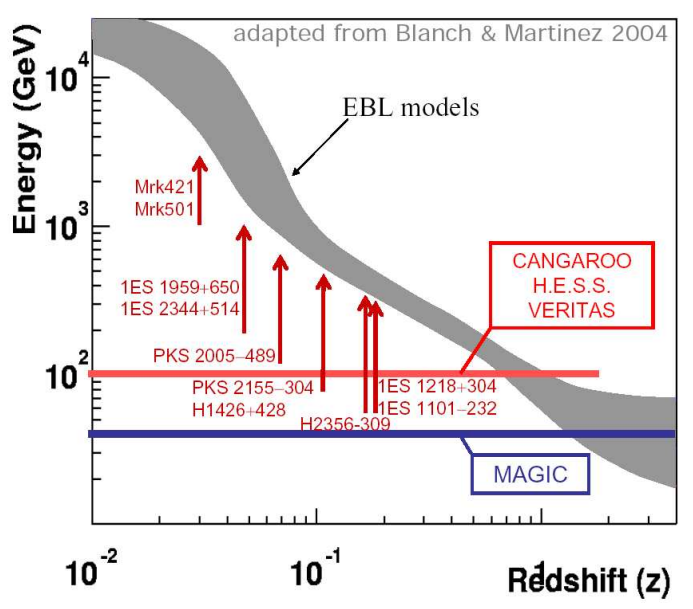

Figure 10: Model predictions for the gamma ray horizon as function of gamma ray energy (shaded band). The arrows indicate the redshifts of the known blazars with highly significant $\mathrm{TeV}$ gamma ray detections. Also indicated is the energy threshold of the new generation of imaging Cherenkov telescopes. Figure adapted from [56]. 

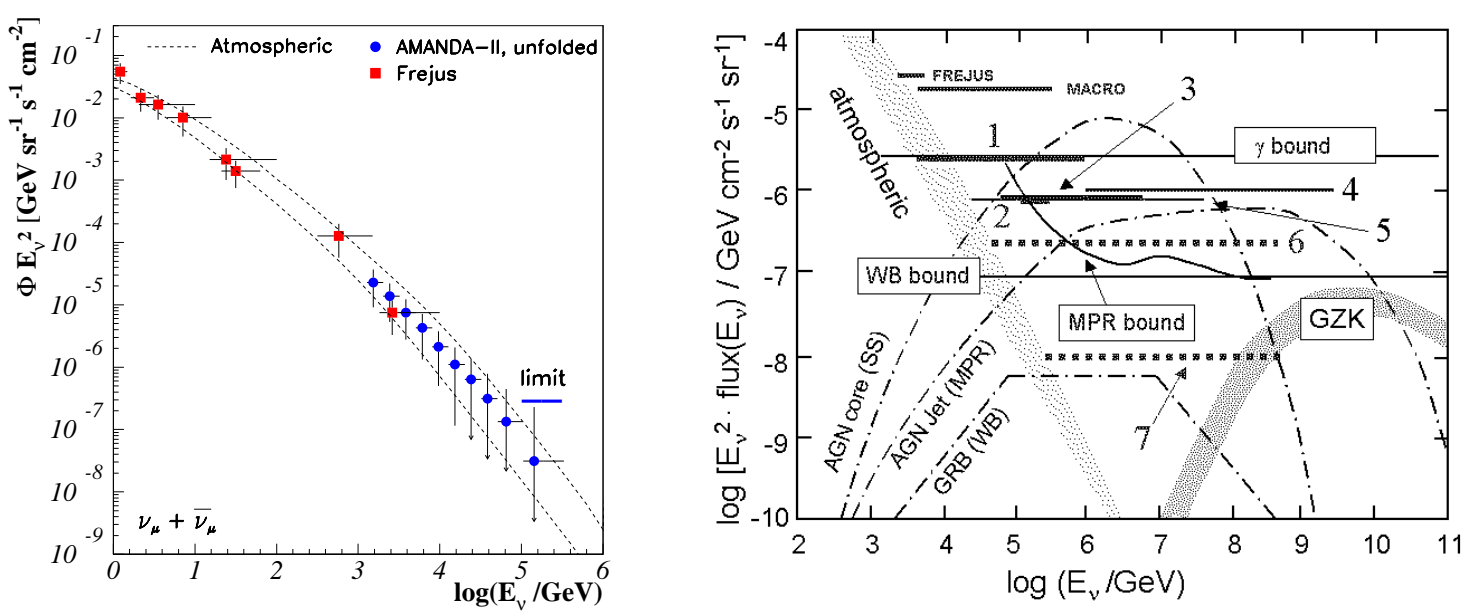

Figure 11: Left: Energy spectrum of muon neutrinos from AMANDA-II and Frejus together with predictions for atmospheric neutrinos (upper line: horizontal fux, lower line: vertical flux). Taken from [60]. Right: Theoretical and experimental limits and model predictions for the diffuse all-fhvour cosmic neutrino flux, assuming a $1: 1: 1$ fhvour mix. Limits 1, 2, 3 and 4 are from AMANDA-B10 and AMANDA-II, limit 5 from the Baikal telescope NT-200, line 6 is the combined AMANDA-II and NT200+ expectation for 4 years and line 7 is the IceCube sensitivity for 3 years. See [61] for further explanations and references.

olds of the Cherenkov telescopes, also indicated in Fig. 10, suggest that redshifts up to $z \approx 0.5$ and higher might be reachable. Indeed the three most distant blazars were discovered very recently by MAGIC [57] and H.E.S.S. [58]. The H.E.S.S. collaboration analyzed the EBL intensity on the basis of the spectra measured for the distant blazars H 2356-309 $(z=0.165)$ and 1ES 1101-232 $(z=0.186)$. Requiring that the unabsorbed source spectra do not become unphysical, an upper limit limit for the EBL intensity is found which is close to the lower end of the allowed range [58]. This surprising result suggests that the Universe is much more transparent to very high energy gamma rays than previously thought.

\section{Neutrinos from cosmic ray sources}

Neutrino astrophysics is currently dominated by the AMANDA-II detector [59] in the antarctic ice and by the smaller neutrino telescope in Lake Baikal. In addition, Mediterranean detectors are in preparation. Future detectors with $\mathrm{km}^{3}$ active volume are under construction or in the planning phase.

For most analyses, up-going muons are used as signatures for muon-neutrinos which have crossed the earth. The neutrino source direction is measured within a few degrees by the reconstructed muon track direction and the energy is well measured by the Cherenkov light yield. The dominating background from down-going atmospheric muons, reconstructed as up-going, can be well controlled by cleaning cuts. Fig. 11 (left) shows the energy spectrum for up-going muon neutrinos $\left(v_{\mu}+\bar{v}_{\mu}\right)$, measured by AMANDA-II and Frejus (taken from [60]). The data are well described by the expected atmospheric neutrino flux so that an upper limit to an additional diffuse $E^{-2}$ cosmic neutrino flux can be derived. This has been done for the last energy bin (100-300 TeV); 


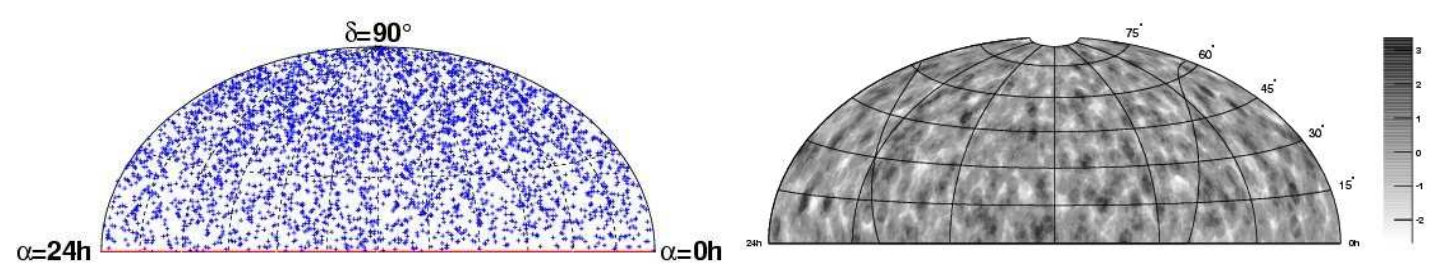

Figure 12: Sky map of $3329 v_{\mu}$ events from AMANDA-II (left) and significance map (right); from [62].

the corresponding $90 \%$ C.L. limit is indicated as a horizontal line. The corresponding limit is also shown in Fig. 11 (right) [61] which summarizes upper limits for the diffuse cosmic flux for all neutrino flavours, assuming that neutrino oscillations produce an even mixture $v_{e}: v_{\mu}: v_{\tau}=1: 1: 1$. Fig 11 (right) also summarizes upper limits in other energy ranges using alternative techniques, the analysis of cascade events in which showers are produced by electron or tau neutrinos and the search for ultra-high energy neutrinos from the horizon or even from above. Various theoretical upper bounds and model predictions are also indicated. It can be seen that some models are already constrained. At the expected sensitivity for the $\mathrm{km}^{3}$ size detector IceCube [59] after 3 years of operation (line 7 in Fig. 11), detection of diffuse neutrinos seems likely.

Higher sensitivities can be reached, adding spatial or temporal constraints. Fig. 12 (left) [62] shows the sky-map of 3329 selected $v_{\mu}$ candidates from AMANDA-II. This map was scanned for localized excesses using highly overlapping search bins of optimized dimensions, the background being estimated from the corresponding full zenith belt. The resulting significance map, Fig. 12 (right), shows a highest excess at $3.35 \sigma$. Taking correlations and trial factors into account, this corresponds to a probability of a background fluctuation of $92 \%$ and is hence not signifi cant. The same sky-map was used to search for excesses in the direction of 33 pre-defi ned source candidates (see [62] for details), again with no signifi cant signals. Typical upper limits for $E^{-2}$ fluxes above $10 \mathrm{GeV}$ are in the order of $10^{-8} \mathrm{~cm}^{-2} \mathrm{~s}^{-1}$. Also after enhancing the sensitivity by source staggering and by searching in time windows of enhanced X-ray or radio emission for special variable sources, no signifi cant signal was found up to now.

An interesting result emerged from the global search for neutrino flares in optimized angular search regions around twelve variable gamma ray and X-ray sources. Clusters of neutrino candidates within 20 days (galactic sources) or 40 days (extragalactic sources) were searched for. Although this search suffers from a trial-factor penalty, it has the advantage that periods without flaring electromagnetic emission are not automatically excluded. No triplets but seven doublets were found. The background probability is larger than $32 \%$ in all cases. When revisiting the time series of neutrino candidates for the blazar 1ES 1959+650, however, it was observed that three of a total of fi ve selected events cluster in a time window of 66 days, see Fig. 13 [62]. This interval happens to overlap partly with a multi-wavelength campaign [64] of gamma ray and X-ray detectors, launched in order to monitor the source which was going through an episode of exceptionally high activity. Interestingly, one of the neutrino candidates was almost coincident with a so-called orphan flare (fi rst vertical line in Fig. 13 (right)), a period of high gamma ray emission, not accompanied by a high X-ray flux. As discussed in section 4, this might be indicative of gamma-rays from high energy hadronic interactions in which also high energy neutrinos should be produced $[65,66]$. However, since this interesting coincidence was discovered a posteriori, i.e. not in a blind anal- 


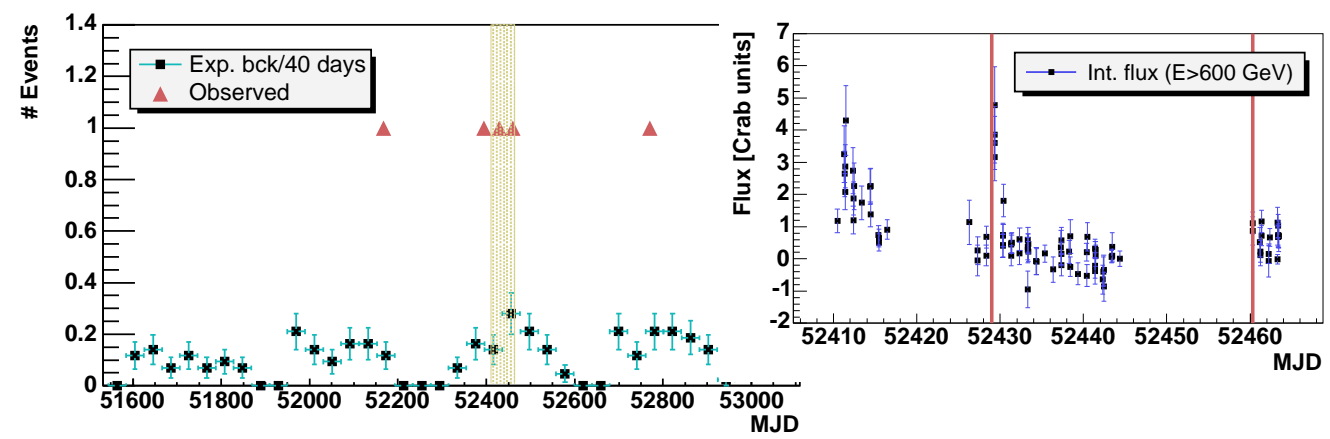

Figure 13: Arrival time distribution of the AMANDA-II neutrino candidates within $2.25^{\circ}$ of the blazar 1ES 1959+650 together with background estimates (left). The shaded area indicates the observation period of the Whipple gamma ray telescope, the results of which are shown on the right [63]. The arrival times of the neutrino events are indicated by vertical lines on the right. Picture taken from [62].

ysis, the AMANDA collaboration assigns a low signifi cance to this observation. Nevertheless, a lesson has been learned, namely that multi-wavelength electromagnetic observations and neutrino observations are by no means independent and should be combined as much as possible, maybe including also other wavelengths and messengers. Such "multi-messenger" strategies [62] might lead to a break-through in neutrino astrophysics before $\mathrm{km}^{3}$ detectors become available.

\section{Summary}

The almost hundred year old puzzle of the origin of cosmic rays still exists but it is under massive pressure due to major progress in astroparticle physics with hadrons, gamma rays and neutrinos. Large air shower arrays are starting to produce a consistent picture for spectra, composition and arrival directions of highest energy cosmic rays, the gamma-ray sky is quickly filling up with new galactic and extragalactic sources, some of them belonging to totally new source classes, and neutrino astronomy may start even before $\mathrm{km}^{3}$ detectors become available using multi-messenger strategies.

Acknowledgments: The author would like to thank E. Bernardini, D. Camin, P. Chadwick, A. Haungs, K.H. Kampert, J. Knapp, J. Ridky, T. Schweizer and C. Spiering for their help in preparing this talk.

\section{References}

[1] J. Cronin, T.K. Gaisser, and S.P. Swordy, Sci. Amer. bf 27644 (1997).

[2] D. Ardouin et al., astro-ph/0504240.

[3] H. Falcke et al., Nature 435313 (2005) [astro-ph/ 0505383 ].

[4] D. Saltzberg, astro-ph/0501364.

[5] A. Haungs, H. Rebel, and M. Roth, Rep. Prog. Phys. 66 (2003) 1145.

[6] T. Antoni et al. (KASCADE Collaboration), Nucl. Instr. Meth. A 513 (2003) 429. 
[7] G. Navarra et al., Nucl. Instr. Meth. A 518 (2004) 207.

[8] T. Antoni et al. (KASCADE Collaboration), Astropart. Phys. in press [astro-ph/0505413].

[9] A. Haungs et al. (KASCADE Collaboration), to appear in Nucl. Phys. B (Proc. Suppl.), Proc. of the XIII ISVHECRI, Pylos (2004) [astro-ph / 0412610 ].

[10] N.N. Kalmykov and S.S. Ostapchenko, Phys. Atom. Nucl. 56 (1993) 346.

[11] R. Engel et al., 26 ${ }^{\text {th }}$ ICRC, Salt Lake City 1 (1999) p.415.

[12] K. Greisen, Phys. Rev. Lett. 16 (1966) 748.

[13] G.T. Zatsepin and V.A. K'uzmin, Pis'ma Zh. Eksp. Teor. Fiz. 4 (1966) 114 [JETP Lett. 4 (1966) 78].

[14] F. Halzen, et al., Astropart. Phys. 3 (1995) 151.

[15] J.W. Elbert and P. Sommers, Astrophys. J. 441 (1995) 151 [astro-ph/9410069].

[16] M. Takeda et al., Astropart. Phys. 19 (2003) 47 [astro-ph/ 0209422 ].

[17] R.U. Abbasi et al. Phys. Lett. B619 (2005) 271 [astro-ph/0501317].

[18] A. Zech, these proceedings.

[19] D.J. Bird et al., Phys. Rev. Lett. 71 (1993) 3401.

[20] Pierre Auger Collaboration, astro-ph/0507150.

[21] J. Abraham et al. (Pierre Auger Collaboration), Nucl. Inst. Meth. A523 (2004) 50.

[22] D. Camin, these proceedings.

[23] M. Teshima et al. (AGASA Collaboration), 28 th ICRC, Tsukuba (2003) 341.

[24] N. Hayashida et al. (AGASA Collaboration), 26 $6^{\text {th }}$ ICRC, Salt Lake City 1 (1999) 353 [astro-ph/9906056].

[25] J.A. Bellido et al., Astropart. Phys. 15 (2001) 167 [astro-ph/ 0009039 ].

[26] R.U. Abbasi et al. (High Resolution Fly's Eye Collaboration), Astrophys. J. 610 (2004) L73 [astro-ph/0404137].

[27] Pierre Auger Collaboration, astro-ph/0507600.

[28] Pierre Auger Collaboration, astro-ph/0507331.

[29] C. Bigongiari, these proceedings.

[30] J. Holder et al. (VERITAS Collaboration), 29 ${ }^{\text {th }}$ ICRC, Pune (2005) [astro-ph / 0507451 ].

[31] H. Kubo et al. (CANGAROO Collaboration), New Astron. Rev. 48 (2004) 323.

[32] J. Hinton et al. (H.E.S.S. Collaboration), New Astron. Rev. 48 (2004) 331 [a stro-ph / 0403052 ].

[33] R.A. Ong, Rapporteur talk, $29^{\text {th }} I C R C$, Pune (2005).

[34] M.A. Malkov and L. O’C Drury, Rep. Prog. Phys. 64 (2001) 429.

[35] H. Muraishi et al., A\& A 354 (2000) L57 [astro-ph/ 0001047 ].

[36] H. Katagiri et al., Astrophys. J. 619 (2005) L163 [astro-ph/ 0412623 ].

[37] F.A. Aharonian et al. (H.E.S.S. Collaboration), Nature 432 (2004) 75 [a stro-ph/ 0411533 ].

[38] D. Berge, $29^{\text {th }} I C R C$, Pune (2005). 
[39] F.A. Aharonian et al. (H.E.S.S. Collaboration), A\&A 437 (2005) L7 [astro-ph/0 05380 ].

[40] N. Komin, $29^{\text {th }} I C R C$, Pune (2005).

[41] Y. Uchiyama et al., PASJ 54 (2002) L73 [astro-ph/ 0208049 ].

[42] W. Voges et al., $A \& A 349$ (1999) 389 [astro-ph/9909315].

[43] Y. Uchiyama et al., A\&A 400567 [astro-ph/0209217].

[44] K. Tsuchiya et al., Astrophys. J. 606 (2004) L115 [astro-ph/ 0403592 ].

[45] K. Kosack et al., Astrophys. J. 608 (2004) L97 [ast ro-ph / 0403422 ].

[46] F. Aharonian et al. (H.E.S.S. Collaboration), A\& A 425 (2004) L13 [astro-ph/ 0408145$].$

[47] J. Hinton et al., $29^{\text {th }}$ ICRC, Pune (2005).

[48] J. Ripken et al., 29 $9^{\text {th }}$ ICRC, Pune (2005).

[49] F.A. Aharonian et al. (H.E.S.S. Collaboration), Science 307 (2005) 1938 [a st ro-ph/ 0504380 ].

[50] F.A. Aharonian et al. (H.E.S.S. Collaboration), Astrophys. J. in press.

[51] S. Funk, A. Lemière et al., 29 $9^{\text {th }}$ ICRC, Pune (2005).

[52] F. Aharonian et al. (H.E.S.S. Collaboartion), Science 309 (2005) 746 [a st ro-ph/ 0508298 ].

[53] F. Aharonian et al. (HEGRA Collaboration), $A \& A$ in press [astro-ph/0501667].

[54] F. Aharonian et al. (H.E.S.S. Collaboartion), A\& A 439 (2005) 1013 [astro-ph/ 0505219 ].

[55] H. Krawczynski, Blazar Variability Workshop II: Entering the GLAST Era, Miami (2005) [astro-ph/0508621].

[56] O. Blanch and M. Martinez, Astropart. Phys. 23 (2005) [ast ro-ph/ 0406061 ].

[57] M. Meyer et al., 29 $9^{\text {th }}$ ICRC, Pune (2005).

[58] F. Aharonian et al., submitted to Nature, astro-ph/0508073.

[59] C. Perez de los Heros, these proceeding.

[60] A. Groß, 40th Rencontres de Moriond on Electroweak Interactions and Unified Theories, La Thuile (2005) [astro-ph/0505278].

[61] C. Spiering, Nobel Symposium on Neutrino Physics, Haga Slott (2004) [astro-ph/0503122].

[62] E. Bernardini et al. (IceCube Collaboartion), Cherenkov 2005, Palaiseau (2005) [astro-ph/0509396].

[63] D. Holder et al., Astrophys. J. 583 (2003) L9.

[64] H. Krawczynski et al., Astrophys. J. 601 (2004) 151 [astro-ph/ 0310158 ].

[65] F. Halzen, Astropart. Phys. 23 (2005) 537 [astro-ph / 0502449 ].

[66] A. Reimer et al., Astrophys. J. in press [astro-ph/0505233]. 